Journal for

.... ImmunoTherapy of Cancer

\title{
Maximizing cancer therapy via complementary mechanisms of immune activation: PD-1 blockade, neoantigen vaccination, and Tregs depletion
}

Anna Morena D'Alise (D) , ${ }^{1}$ Guido Leoni, ${ }^{1}$ Maria De Lucia, ${ }^{1}$ Francesca Langone, ${ }^{1}$ Linda Nocchi, ${ }^{1}$ Fabio Giovanni Tucci, ${ }^{1}$ Elisa Micarelli, ${ }^{1}$ Gabriella Cotugno, ${ }^{1}$ Fulvia Troise, ${ }^{1}$ Irene Garzia, ${ }^{1}$ Rosa Vitale, ${ }^{1}$ Veronica Bignone, ${ }^{1}$ Elena Di Matteo, ${ }^{1}$ Rosa Bartolomeo, ${ }^{1}$ Deborah H Charych, ${ }^{2}$ Armin Lahm, ${ }^{1}$ Jonathan Zalevsky, ${ }^{2}$ Alfredo Nicosia, ${ }^{1,3}$ Elisa Scarselli ${ }^{1}$

\section{ABSTRACT}

Background A number of different immune pathways are involved in the effective killing of cancer cells, collectively named as the 'Cancer Immunity Cycle'. Anti-PD-1 checkpoint blockade (CPB) therapy is active on one of these pathways and reinvigorates anticancer $T$ cell immunity, leading to long-term responses in a limited fraction of patients with cancer. We have previously shown that neoantigens-based adenovirus vectored vaccine in combination with anti-PD-1 further expands pre-existing anticancer immunity and elicits novel neoantigen-specific T cells thereby increasing efficacy to $50 \%$ of tumor clearance in mice. Here we added a third component to the CPB plus vaccine combination, which is able to modify the suppressive tumor microenvironment by reducing the number of tumor-infiltrating regulatory $T$ cells (Tregs), as strategy for improving the therapeutic efficacy and overcoming resistance.

Methods The antitumor efficacy of anti-PD-1, neoantigen vaccine and Treg modulating agents, either Bempegaldesleukin (BEMPEG: NKTR-214) or an antiCTLA-4 mAb with Treg-depleting activity, was investigated in murine tumor models. We evaluated tumor growth in treated animals, neoantigen-specific T cells in tumors, tumor-infiltrating lymphocytes (TILs) and intratumoral Tregs.

Results The addition of BEMPEG or anti-CTLA-4 to the combination of vaccine and anti-PD-1 led to complete eradication of large tumors in nearby $100 \%$ of treated animals, in association with expansion and activation of cancer neoantigen-specific T cells and reduction of tumorinfiltrating Tregs.

Conclusion These data support the notion that the integrated regulation of three steps of the cancer immunity cycle, including expansion of neoantigen-specific T cells, reversal of the exhausted T cell phenotype together with the reduction of intratumoral Tregs may represent a novel rationally designed drug combination approach to achieve higher cure rates.

\section{BACKGROUND}

Over time, many strategies to induce potent and effective tumor-specific $\mathrm{T}$ cell responses have been devised, including cytokine-based therapies, immune checkpoint blockade and cancer vaccination.

Among cytokines, interleukin-2 (IL-2) (Proleukin) was the first immunotherapy drug approved for clinical use in metastatic renal cell carcinoma and melanoma. IL-2 has very limited use because of the low rate of response and the severe toxicities associated with the treatment. ${ }^{1}$ In addition, IL-2 has some drawbacks in its capability to expand T effector cells and suppressive Tregs, which in turn dampen antitumor immunity. ${ }^{2}$ Bempegaldesleukin (BEMPEG: NKTR-214) is an investigational CD122-preferential IL-2 pathway agonist that leverages the clinically validated IL-2 pathway to stimulate an antitumor immune response. ${ }^{3}$ It consists of recombinant human IL-2 bound to an average of six releasable polyethylene glycol chains. ${ }^{4}$ The molecule's design provides improved pharmacokinetics and pharmacodynamics in comparison with IL-2, while also reducing its binding to IL-2R $\alpha$ (CD25) constitutively expressed on Tregs. ${ }^{45}$ BEMPEG is designed to rapidly expand $\mathrm{CD} 8^{+} \mathrm{T}$ and natural killer (NK) cells without unwanted expansion of Tregs in the tumor microenvironment. Moreover, differently from the first generation IL-2, BEMPEG can selectively deplete intratumoral Tregs. This effect has been demonstrated across different tumor models as well as in patients, suggesting that BEMPEG can play an important role in the modulation of the tumor microenvironment by targeting specific immunosuppressive cells. ${ }^{6}$

Checkpoint blockade (CPB) and, in particular, the blockade of PD-1/PD-L1 interaction is now an established treatment in many 
indications resulting in a long-term cure of cancer in a fraction of patients. ${ }^{78}$ Relief of $\mathrm{T}$ cells from the inhibitory signal mediated by immune checkpoint inhibitors has been shown to improve the response rate. ${ }^{9}$ The effectiveness of this approach relies on the rescue of $\mathrm{T}$ cells against tumor antigens that are spontaneously induced by the tumor. Recently, mutation-associated neoantigens (nAgs) have been demonstrated to be the dominant targets of such spontaneous antitumor $\mathrm{T}$ cell responses. In this scenario, cancer vaccines targeting tumor mutations could uniquely contribute by boosting tumor-induced $\mathrm{T}$ cells and by priming novel $\mathrm{T}$ cells against additional cancer nAgs thereby increasing the potency and breadth of antitumor immunity. Vaccine contribution within this context is very selective to antigens that are present exclusively in the tumor and therefore is expected to be devoid of side effects. Several preclinical studies have already shown that cancer vaccines targeting tumor nAgs can be effective in a variety of murine tumor models, ${ }^{1011}$ supporting the initiation of several clinical studies. ${ }^{12} 13$ Most of these studies are based on the combination of nAg-based vaccines with anti-PD-1/PD-L1 treatment since preclinical data clearly demonstrated that cancer vaccination is not effective as a stand-alone treatment in the presence of high tumor burden. ${ }^{14}$

Replication deficient adenoviruses are very efficient inducers of potent $\mathrm{CD} 8^{+} \mathrm{T}$ cell responses as demonstrated in both mice and primates and, most importantly, in clinical studies, ${ }^{15}$ and therefore represent an ideal choice for the induction of effective antitumor immunity, especially $\mathrm{CD} 8^{+} \mathrm{T}$ cell responses.

Among them, non-human great apes derived adenoviruses (GAds) are optimal vaccine vector candidates because they can overcome the issue of pre-existing human adenovirus neutralizing antibodies that can impair vaccine efficacy. ${ }^{16}$ GAds are clinically validated and proven to be safe in clinical trials of candidate vaccines against hepatitis C, HIV, RSV, malaria and Ebola. ${ }^{17-20}$ We have recently reported that vaccination with GAd encoding multiple nAgs generated powerful $\mathrm{nAg}$-specific $\mathrm{T}$ cell responses in mice and that the combination of the vaccine with anti-PD-1 is effective in eradicating large tumors. ${ }^{14}$ However, despite significant improvement over CPB monotherapy, the combined therapy was successful in curing only half of the treated mice, leaving room for therapeutic improvement. ${ }^{14}$

One rationale behind the success of combination therapies lies in their ability to effectively influence biological regulatory circuits by targeting multiple steps of the cancer immunity cycle to provide incremental benefits. In the present study, we demonstrated that to exploit the full power of vaccination against tumors, combination therapies are needed. In particular, only by blocking the PD-1/PD-L1 axis and reprogramming the tumor microenvironment by BEMPEG, we could achieve tumor regression in $100 \%$ of vaccinated animals. Modulation of the same key molecular switches by using a different therapeutic approach combining a GAd vaccine, an anti-PD-1 antibody and anti-CTLA-4 antibody, supported the mechanism of action required to achieve effective tumor response in a majority of the treated mice. These results suggest that the targeting of different steps of the cancer immunity cycle is crucial for therapeutic outcomes and can reverse resistance to immunotherapy, offering insight into the immunological mechanisms that drive improved antitumor response.

\section{MATERIALS AND METHODS \\ Mice}

Six-week-old female BALB/c or C57BL/6 mice were purchased from Envigo.

\section{Cell lines}

CT26 (BALB/c mouse undifferentiated colon carcinoma) and MC38 (C57BL/6 mouse colon adenocarcinoma) were purchased from ATCC. Cell lines were cultured in complete RPMI-1640 or Dulbecco's modified Eagle medium, respectively, supplemented with $10 \%$ fetal bovine serum, $2 \mathrm{mM}$ L-glutamine, $1 \%(\mathrm{v} / \mathrm{v})$ penicillin/ streptomycin and maintained at $37^{\circ} \mathrm{C}$ in $5 \% \mathrm{CO} 2$.

\section{GAd vaccine}

CT26 neoantigens prediction and GAd vaccine generation are described in D'Alise et $_{\text {al. }}{ }^{14}$

\section{In vivo treatments}

The $2 \times 10^{5}$ CT26 (early tumor setting) and $2 \times 10^{6}$ CT26 or $2 \times 10^{5}$ MC38 (established tumor setting) cells were injected subcutaneously. Treatments were started 3 days later or when large established tumors occurred, respectively. In therapeutic settings treating established tumors, 7-8 days after tumor challenge animals were randomized according to their tumor volumes (tumor size average per group $70-100 \mathrm{~mm}^{3}$ ) prior to treatment. In both settings, GAd vaccine was administered at day 0 (first day of treatment) via intramuscular injection, at $5 \times 10^{8}$ viral particles (vp), by delivering a volume of $50 \mu \mathrm{L}$ per quadriceps. BEMPEG, supplied by Nektar Therapeutics, was administered at $0.8 \mathrm{mg}$ per $\mathrm{kg}$, diluted in BEMPEG buffer and injected by intravenous injection, according to previous studies. ${ }^{4}$ In early therapeutic setting studies, the first BEMPEG treatment was concomitant (day 0) or sequential (day 7 ) to GAd vaccination, then repeated every 7 days $\times 3$, whereas in the efficacy studies with CPI, BEMPEG treatment was initiated at day 7 , then repeated every 7-9 days $\times 3$. $\alpha-m P D-1 \quad(200 \mu g$, BioXcell, clone RMP114) was administered twice a week until day 16 , whereas $\alpha$-mCTLA-4 9D9_IgG2A (100 $\mu$ g, InvivoGen) was administered once at day 0 . Both antibodies were intraperitoneally injected. Tumor growth was measured using a digital caliper every 3-4 days. Tumor volume was calculated using the formula: $0.5 \times$ length $\times$ width $^{2}$, where the length was the longer dimension. Mice were sacrificed immediately on signs of distress or a tumor volume above $1500 \mathrm{~mm}^{3}$ occurred. 
Mice that were completely cured received a second tumor challenge $\left(2 \times 10^{5}\right.$ MC38 on the contralateral left flank) at day 100 . Tumor growth was monitored over time until day 300 .

\section{Ex vivo immune analysis}

Intracellular interferon-gamma (IFN- $\gamma$ ) staining was performed on splenocytes or isolated TILs on stimulation with peptides pool at a final concentration of $2 \mu \mathrm{g} / \mathrm{mL}$ for each peptide, in the presence of Golgi plug (catalog 555029, BD Biosciences). Dimethyl sulfoxide (catalog D2650, Sigma-Aldrich) and phorbolmyristate acetate/ ionomycin (Sigma-Aldrich) were used as negative or positive controls. After overnight $(\mathrm{ON})$ stimulation, cells were incubated with purified anti-mouse CD16/CD32, clone 2.4G2 (BD Biosciences) and then stained in FACS buffer (phosphate-buffered saline, $1 \%$ fetal calf serum) with the following surface antibodies: allophycocyanin antimouse CD3e: clone 145-2 C11; phycoerythrin anti-mouse CD4: clone L3T4 and PerCP anti-mouse CD8a: clone 53-6.7 (all from BD Biosciences). Intracellular staining was performed after treatment with Cytofix/Cytoperm (catalog 51-2090Kz) and in the presence of PermWash (catalog 554723) (both from BD Biosciences) using fluorescein isothiocyanate antimouse IFN- $\gamma$ (clone XMG1.2, BD Biosciences). Stained cells were acquired on a FACS Canto flow cytometer and analyzed using DIVA software (BD Biosciences). At least $20000 \mathrm{CD}^{+}, \mathrm{CD}^{+}$gated events were acquired for each sample.

Tregs staining was performed on single-cell suspensions from tumors. After a previous incubation with purified anti-mouse CD16/CD32, clone 2.4G2, cells were stained with Live/Dead Fixable Near-IR Dead Cell stain kit (Life Technologies). Surface antigen staining was then performed in FACS buffer with the following antibodies: PB anti-mouse CD4: clone RM4-58 (Biolegend), BV510 antimouse CD8a: clone 53-6.7 (Biolegend) and PerCPCyanine 5.5 anti-mouse CD45: clone: 30-F11 (eBioscience). Following ON cells fixation and permeabilization (performed by using Foxp3 staining buffer set, eBioscience), Foxp3 intracellular staining was performed with APC anti-mouse Foxp3, clone FJK-16S (eBioscience). Stained cells were acquired on a FACS Canto flow cytometer and analyzed using DIVA software (BD Biosciences).

\section{TILs preparation for IFN- $\gamma$ intracellular cytokine staining (ICS)}

Isolated tumors were first dissociated and digested with collagenase I (Gibco) at $37^{\circ} \mathrm{C}$ for 2 hours. Tumor homogenates were depleted from erythrocytes using ACK lysing buffer (Gibco) and filtered through $70 \mu \mathrm{m}$ cell strainers to generate single-cell suspensions. TILs were isolated from tumor homogenates (Pan T cells isolation kit II, Miltenyi Biotec) and cocultured with APC from spleen of naïve mice in presence of antigen stimuli.

\section{Gene expression analysis}

NGS was performed at Genomix4Life S.r.l (Salerno). RNA extracted from tumor samples was fragmented, and sequencing libraries were prepared using IlluminaTruSeq mRNA stranded kit. Sequencing was performed at target depth of 60 million of paired-end reads. Raw NGS reads were aligned on mm10 genome by using hisat2 software. Differentially expressed genes (DEGs) were determined by using four alternative methods: Deseq2, EdgeR, limma with Voom correction and NOISeq. Each method was run on a count matrix reporting the number of reads mapped to each gene by the Rsubread package. For each comparison, we retained only genes identified by the consensus of three out of the four methods, with a difference of $\log 2 \mathrm{FC}$ of at least \pm 1 and a Benjamini-Hochberg corrected $p$ value $\leq 0.05$. Finally, DEGs with $<10$ total count of mapped reads and expressed with a mean TPM $<1$ were filtered out.

\section{Statistics}

Statistical significance was determined by GraphPad Prism using the nonparametric, two-tailed Mann-Whitney $\mathrm{U}$ test or as otherwise stated in the figure legend.

\section{RESULTS}

\section{BEMPEG synergizes with GAd vaccine when administered after vaccination}

The antitumor effect of BEMPEG combined with a GAd vaccine was evaluated in a murine tumor model. A multiantigenic GAd vaccine encoding 31 CT26 nAgs (GAd-CT26-31) was generated based on MHC class I and class II binding predictions, tumor allele frequency and RNA expression, as previously described. ${ }^{14}$ The combination of the GAd-CT26-31 $\mathrm{nAg}$ vaccine with BEMPEG was tested on CT26 tumors in an early therapeutic setting. Aiming to find the optimal vaccine and BEMPEG schedule, different regimens of treatment were tested. BALB/c mice were implanted subcutaneously with CT26 cells and 3 days later, concomitant (GAd and BEMPEG at day 0) or sequential administration (GAd day 0 and BEMPEG day 7) of vaccine and BEMPEG were started, according to the scheme depicted in figure 1A. Simultaneous delivery of GAd and BEMPEG at day 0 was not effective to control tumor growth, and none of the animals was tumor free after treatment. In contrast, administration of the GAd vaccine at day 0 and BEMPEG on day 7 resulted in a strong antitumor activity, with $70 \%$ of treated mice assessed as tumor free (figure 1B). Monotherapies with GAd or BEMPEG were also evaluated, demonstrating poor efficacy of both vaccine and BEMPEG as a stand-alone treatment when given at day 0 or at day 7 (figure 1B). The levels of immune responses induced in mice receiving concomitant versus sequential GAd and BEMPEG treatment were also evaluated. $\mathrm{CD}^{+}$and $\mathrm{CD}^{+}$nAg-specific $\mathrm{T}$ cell responses were measured on splenocytes restimulated with a pool of peptides corresponding to each neoepitope inserted in the vaccine construct. Concomitant treatment with GAd and BEMPEG at day 0 resulted in a weak $\mathrm{nAg}$-specific $\mathrm{T}$ cell response, consistent with a poor therapeutic efficacy 

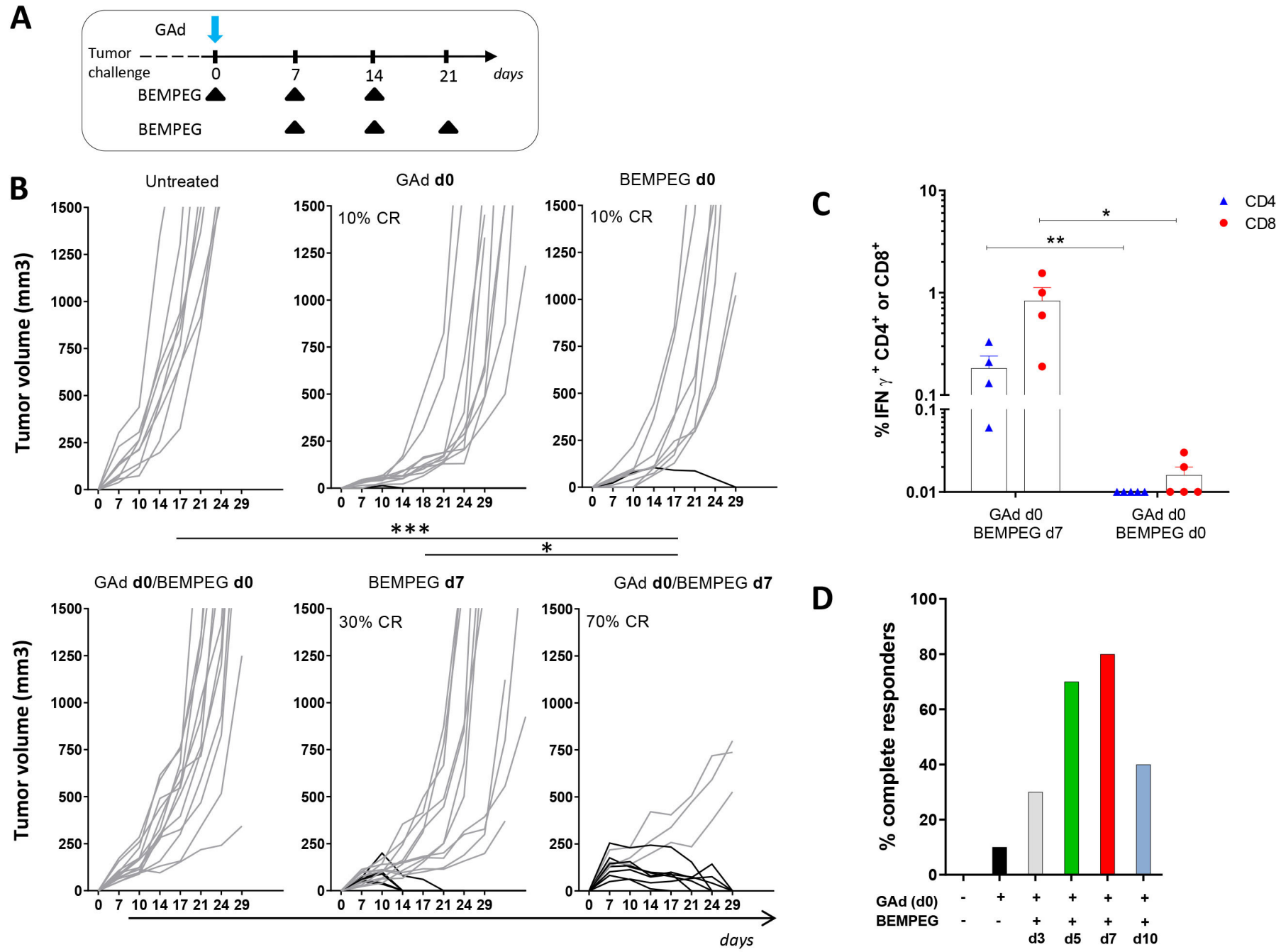

Figure 1 Sequential BEMPEG treatment synergizes with GAd-based vaccine. (A) Schematic of treatments. BALB/c mice were injected subcutaneously with CT26 tumor cells and 3 days later treated with GAd and/or BEMPEG. The first BEMPEG administration was concomitant (day 0) or sequential (day 7) to GAd, then repeated every 7 days as indicated. (B) Tumor volume $\left(\mathrm{mm}^{3}\right)$ over time. Each line represents an individual mouse $(\mathrm{n}=9-16)$ (gray lines for non-responders and black lines for responders). Percentages on the graphs indicate the rate of response as sum of complete and partial response ( $\geq 40 \%$ tumor shrinkage). (C) Percentages of IFN $-\gamma^{+} \mathrm{CD}^{+}$or $\mathrm{CD}^{+} \mathrm{T}$ cells upon peptide stimulation measured in splenocytes from $\mathrm{GAd} /$ BEMPEG day0 or GAd/BEMPEG day7 treated mice. ICS was performed 2 weeks after vaccination. Bars show mean+SEM . Each symbol represents an individual sample, $n=4-5$ (blue: CD4 ${ }^{+}$T cells; red: $C D 8^{+} T$ cells). Data in figure parts $B$ and $C$ are representative of at least two independent experiments. Statistics were generated using mid-p exact test $\left({ }^{\star \star *} p \leq 0.001,{ }^{* \star} p \leq 0,01\right.$, ${ }^{*} \mathrm{p} \leq 0.05$ ). (D) Percentage of complete responders to GAd in combo with BEMPEG given at different time-points (d3, d5, d7 or d10) from vaccination. Data were pooled from two independent experiments. GAd, great apes derived adenovirus.

for this dosage regimen (figure 1C). On the contrary, strong responses were observed in mice receiving vaccine at day 0 and BEMPEG at day 7 in agreement with previous data showing that IL-2 administration is particularly effective during the $\mathrm{T}$ cell activation phase. ${ }^{21}$ The interval between GAd and BEMPEG administration was further dissected, exploring the time window between GAd vaccine at day 0 and BEMPEG given at day 3, 5, 7 or 10 -. The results confirmed the interval of 7 days as optimal to achieve the best effect (figure 1D). The treatments with BEMPEG at 5 or 7 days were not statically different.
Combination of GAd, BEMPEG, and anti-PD-1 results in $100 \%$ complete regression of large tumors

Having set the optimal schedule for GAd and BEMPEG administration, we sought to confirm their synergistic effect in a stringent model of large established tumors. Our previous work has shown that the therapeutic outcome of GAd vaccination can be very different depending on the tumor setting used. Moreover, immunotherapies that are effective on early treatments might fail in situations of advanced disease, given the highly immunosuppressive tumor microenvironment. ${ }^{14}$ In particular, we have demonstrated that effective antitumor response in the presence of a high tumor burden requires concomitant 
A

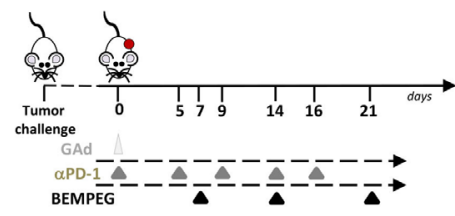

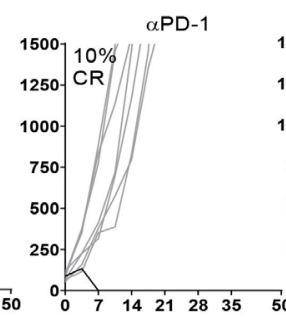

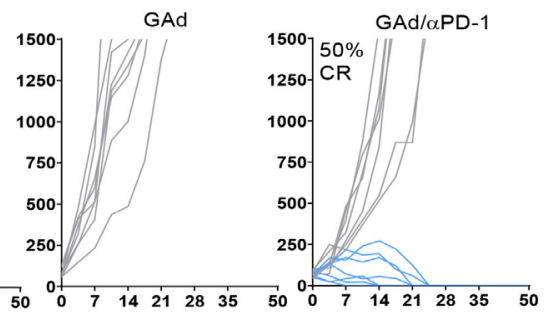

C
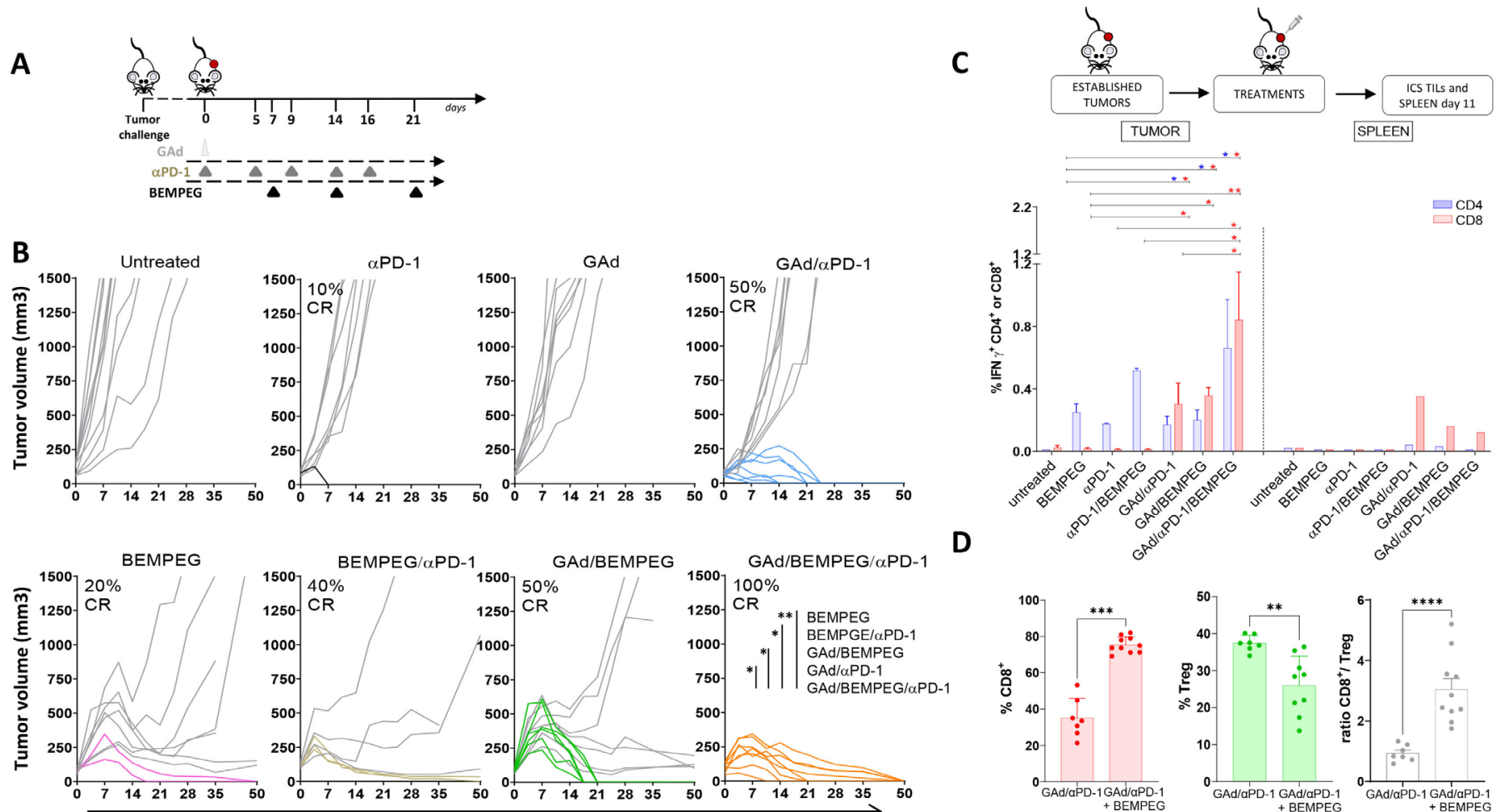

GAd/BEMPEG/ $\alpha$ PD-1

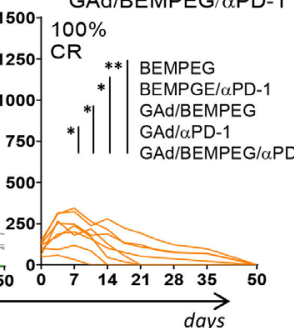

Figure 2 The triple combination GAd/BEMPEG/oPD-1 results in 100\% efficacy in association with intratumoral expansion of vaccine-induced $\mathrm{nAg} T$ cells and tumor microenvironment (TME) reshaping. (A) Experimental scheme. Randomized BALB/c mice bearing 7-day-old subcutaneous CT26 tumors were treated with monotherapies (GAd, BEMPEG or $\alpha$ PD-1), double combo

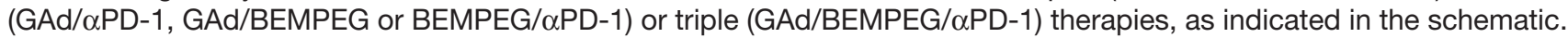
(B) Tumor volume $\left(\mathrm{mm}^{3}\right)$ over time. Each line represents an individual sample (gray lines for non-responders and colored lines for responders). Percentages on the graphs indicate the rate of complete responses (CR). Data ( $n=7-14$ mice/group) are representative of at least two independent experiments. Statistics were generated using mid-p exact test $\left({ }^{\star *} p \leq 0.01 ;{ }^{*} p \leq 0.05\right)$. (C) Immune response analysis was performed by ICS in TILs versus spleen harvested at day 11. BALB/c mice bearing established subcutaneously CT26 tumors were treated with $\alpha$ PD-1 or BEMPEG as monotherapies, with double combo $\alpha$ PD-1/BEMPEG, GAd/ $\alpha$ PD-1, GAd/BEMPEG or triple combo GAd/ $\alpha$ PD-1/BEMPEG. Untreated mice as negative control. Percentages of IFN- $\gamma$ ${ }^{+} \mathrm{CD} 4^{+}$or $\mathrm{CD} 8^{+} \mathrm{T}$ cells specific to the pool of vaccine-encoded neo-peptides are shown. Bars show mean+SEM (blue bars: $\mathrm{CD}^{+}$; red bars: $\left.\mathrm{CD}^{+}\right)$. Individual samples $(\mathrm{n}=2-6)$ for tumor or a pool of samples for spleen were analyzed. Data were pooled from two independent experiments. Statistics were generated using unpaired Mann-Whitney non-parametric test $\left({ }^{\star *} \mathrm{p} \leq 0.01\right.$; ${ }^{*} \mathrm{p} \leq 0.05$ ). (D) Percentages of $\mathrm{CD}^{+}$(red bars) T cells or Tregs (green bars) and ratio CD8 ${ }^{+} /$Treg (white bars) in the tumor of GAd/ $\alpha P D-1$ versus GAd/ $\alpha$ PD-1/BEMPEG treated mice. Bars show mean+SEM; each symbol represents an individual sample. Data ( $n=7-10$ mice/group) were pooled from two independent experiments. Statistics were generated using unpaired Mann-Whitney non-parametric test $\left({ }^{\star \star \star \star} p \leq 0.0001 ;{ }^{* \star \star} p \leq 0.001,{ }^{*} p \leq 0.05\right)$. GAd, great apes derived adenovirus; nAg, neoantigen.

treatment with GAd vaccine and checkpoint inhibitors such as anti-PD-1. Therefore, the antitumoral effect of GAd/BEMPEG was evaluated in mice bearing large tumors, in the presence or absence of anti-PD-1. CT26 cells were injected subcutaneously, and tumors were grown to $100 \mathrm{~mm}^{3}$ prior to treatment. At this time (day $0)$, mice were vaccinated with GAd, and a week later, they received BEMPEG, when some tumors already reached a volume above $200 \mathrm{~mm}^{3}$. The dosing of BEMPEG was repeated every week according to the regimen illustrated in figure $2 \mathrm{~A}$.

BEMPEG monotherapy demonstrated modest efficacy, resulting in $20 \%$ of cured animals. Vaccination was completely ineffective as a monotherapy. In contrast, combined treatment with GAd and BEMPEG induced tumor regression in $50 \%$ of mice (figure 2B), similar to the response rate achieved by combining GAd and
anti-PD-1 (figure 2B and ref 14). Synergistic activity resulting in complete tumor regression in $40 \%$ of treated animals was also observed when combining BEMPEG with anti-PD-1, in line with recent studies. ${ }^{6}$ Interestingly, in the three groups treated with BEMPEG-based dual combinations, we observed initial reduction of tumor volumes followed by stabilization of the volume in some of the mice and uncontrolled tumor growth in others. Triple combination therapy with GAd/BEMPEG/ anti-PD-1 exhibited the most effective tumor inhibition, demonstrating complete cure in $100 \%$ of treated animals (figure 2B). To interrogate the mechanisms of how the triple therapy worked, we measured the levels of antigen-specific $\mathrm{T}$ cells in the tumors of treated mice after ex vivo restimulation with vaccine-encoded cognate neoantigenic peptides, 11 days following commencement of treatments. Using flow cytometry, we observed 
significant expansion of intra-tumoral $\mathrm{CD}^{+}$vaccineinduced $\mathrm{T}$ cells in the triple combination group, GAd/ BEMPEG/anti-PD-1, as compared with dual or single agent treatments (figure 2C), including the previously reported combination of GAd and anti-PD- $1 .{ }^{14}$ Consistent with the expansion of vaccine-induced antigen specific $\mathrm{T}$ cells, the addition of BEMPEG to the GAd and antiPD-1 regimen elicited a strong increase of intratumoral $\mathrm{CD}^{+} \mathrm{T}$ cells, with higher numbers of $\mathrm{CD}^{+} \mathrm{T}$ cells infiltrating the tumor in the triple therapy group compared with GAd/anti-PD-1 treated mice ( $75 \%$ vs $35 \%$, respectively) (figure 2D). In addition, treatment with the triple combination induced reduction of immunosuppressive intratumoral Tregs, with a significant shift of the immune balance in the tumor microenvironment in favor of CD8 antitumor immunity over Tregs indicated by increased $\mathrm{CD} 8^{+} /$Tregs ratio (figure 2D).

\section{Combination of GAd, BEMPEG, and anti-PD-1 increases} expression of immune activation genes in the tumors

To gain insight into the immune mechanism underlying the strong therapeutic effect achieved by the triple combination of GAd/BEMPEG/anti-PD-1 in the established tumor setting, we performed RNA sequencing (RNA-seq) analysis of tumors. Samples were harvested at day 13 from responder mice showing tumor shrinkage belonging to the following groups: (1) BEMPEG, (2) anti-PD-1, (3) antiPD-1/BEMPEG, (4) GAd/BEMPEG, (5) GAd/anti-PD-1, and (6) GAd/BEMPEG/anti-PD-1. As control, tumors from untreated mice were also harvested and used to identify DEGs in each experimental group. Mice treated with the vaccine alone were also analyzed and showed a transcription profile indistinguishable from untreated mice (figure $3 \mathrm{~A}$ ), consistent with the lack of tumor shrinkage as previously described. ${ }^{14}$ Hierarchical clustering of the most variable genes demonstrated that the major changes in gene expression compared with the untreated mice were induced on triple combination treatment, followed by BEMPEG treatment and GAd/anti-PD-1 (figure 3A and online supplemental figure 1A). Indeed, nearly all of the genes modulated in response to anti-PD-1 therapy were also found in the GAd/anti-PD-1 group and the triple combination group, reflecting a common effect of antiPD-1 on tumor profiles (online supplemental figure 1C). Distinct from anti-PD-1 monotherapy, BEMPEG activated

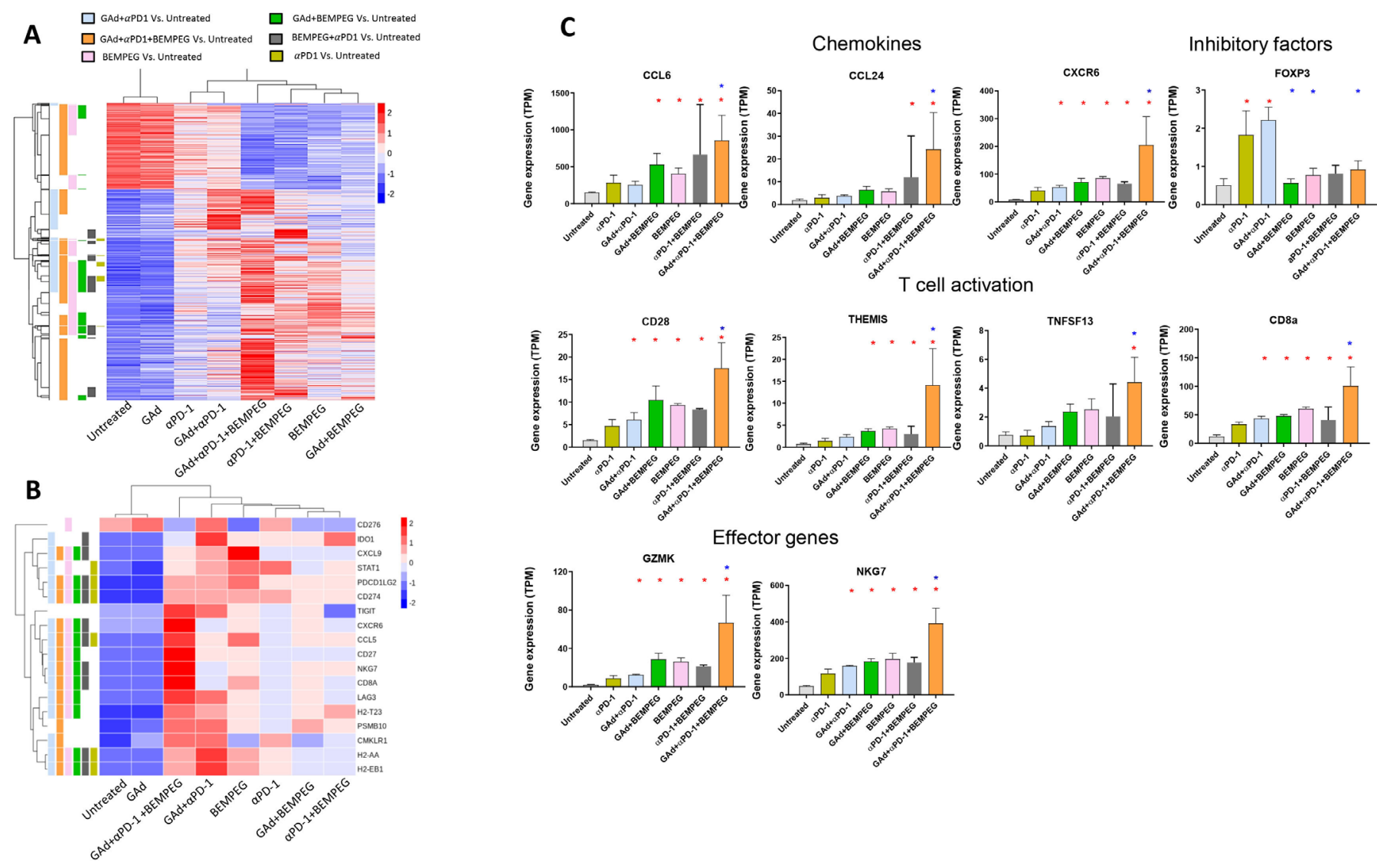

Figure 3 Combination of GAd, BEMPEG and anti-PD-1 increases expression of immune-related genes in the tumors. (A) Heatmap representing the differentially expressed genes (red: upregulated; blue: downregulated) detected by RNAseq on tumors of responder mice for each group of treatment versus untreated ( $n=3-5 /$ group) (median log2 fold change FC $<-1$ or $>1$; Benjamini-Hochberg corrected $p$ value $<0.05$ ). (B) Heat map of the 18 genes belonging to a tumor inflammation signature that predicts response to anti-PD-1 across multiple solid tumors. ${ }^{22}$ (C) Bar charts of select T cell genes. Genes found differentially expressed when compared with untreated are indicated by a red asterisk, while the blue asterisk indicates genes differentially expressed when compared with GAd/ $\alpha$ PD-1. GAd, great apes derived adenovirus. 
a much larger number of genes, with a discrete number of them exclusively present in monotherapy (figure 3A and online supplemental figure 1B). Higher enrichment in the number of genes specifically modulated by GAd/ BEMPEG/anti-PD-1 was always observed compared with each double treatment regimen. Importantly, all treatments associated with an effective therapeutic response induced a marked upregulation of genes belonging to a tumor inflammation signature that has been shown to be predictive of a clinical response in patients treated with anti-PD-1 (figure 3B). ${ }^{22}{ }^{23}$ Again, the most pronounced effect was observed in the GAd/anti-PD-1/BEMPEG group, with a significant upregulation of several genes compared with all other groups (figure 3B,C). The added value of BEMPEG on the combination of GAd/anti-PD-1 is linked to the upregulation of chemokines and their receptors in tumors of mice treated with GAd/anti-PD-1/ BEMPEG versus GAd/anti-PD-1, including CCL6, CCL24 and CXCR6, previously reported to be associated with the presence of effector $\mathrm{T}$ cells in the tumor site. ${ }^{24}$ Expression of key genes associated with costimulatory and effector function of $\mathrm{T}$ cells (CD28, Themis,Granzyme $\mathrm{K}, \mathrm{NKG7}$ ) was also substantially increased in the triple combination compared with GAd/anti-PD-1 (figure 3C). These results support enhanced expression of an array of important molecules sustaining intratumoral recruitment as well as function of $\mathrm{T}$ cells in the triple combination group. Finally, transcriptomic data showed an important downregulation of intratumoral Foxp3 in response to BEMPEG, consistent with the effect of intratumoral Tregs depletion by BEMPEG recently reported. ${ }^{6}$

\section{Effective reprogramming of tumor microenvironment by anti-CTLA-4 in combination with vaccine and anti-PD-1 recapitulates the maximal efficacy of GAd/anti-PD-1 and BEMPEG}

The outstanding immunotherapeutic effects of the triple combination correlates with increased expansion of antigen-specific $\mathrm{T}$ cells elicited by the GAd vaccine accompanied by an increase of tumor infiltrating $\mathrm{CD} 8^{+}$ $\mathrm{T}$ cells and reduction of Tregs in mice receiving GAd, anti-PD-1 and BEMPEG. Therefore, this combination has the potential to adjust the immunosuppressive TME promoting a positive shift of the immunosuppressive TME into an immune-active phenotype. To corroborate the mechanisms of how the triple therapy works, we exploited a second approach based on the combination of the GAd vaccine, anti-PD- 1 and anti-CTLA-4. Anti-CTLA-4 mAbs have been extensively studied in mouse models of cancer, where the therapeutic effect on established tumors relies on the effect of anti-CTLA-4 in promoting the expansion of T effector cells and reduction of Treg cells at the tumor site, resulting in an increase in intratumoral Teff/Tregs cell ratio. ${ }^{25}$ Antitumor activity of GAd, anti-PD-1 and anti-CTLA-4 was evaluated in the CT26 mouse model, under the same experimental conditions in which GAd/ anti-PD-1/BEMPEG treatment was tested, compared with single or dual therapies (figure 4A). Similar to the effect of the triple combination, GAd/anti-PD-1/BEMPEG, the triple therapy GAd/anti-CTLA-4/anti-PD-1 led to regression of large tumors in $90 \%$ of treated mice with superior effect over the mono and dual therapies (figure 4A). Interestingly, the addition of anti-CTLA- 4 to the dual therapy GAd and anti-PD-1 previously employed ${ }^{14}$ promoted selective reduction of intratumoral Tregs along with concomitant expansion of $\mathrm{CD}^{+} \mathrm{T}$ cells in the tumor, resulting in an overall increase of intratumoral Teff/Tregs ratio of about fourfold (figure 4B). These data underline a common mechanism required to overcome therapeutic resistance for improved antitumor response and highlights the intratumoral ratio of Teff/Tregs as a relevant biomarker of treatment efficacy.

\section{Effectiveness of the triple combination GAd, anti-PD-1, and BEMPEG is confirmed in a second tumor model}

The efficacy of the triple combination therapy, GAd/antiPD-1/BEMPEG, was investigated in the MC38 cell line derived from C57BL/6 murine colon adenocarcinoma. For these experiments, we used a GAd vaccine encoding seven nAgs previously identified in MC38 cells by mass spectrometry analysis and shown to induce $\mathrm{CD}^{+} \mathrm{T}$ cell responses. ${ }^{11}{ }^{14}$ In this model, vaccination is not effective as a stand-alone treatment, and monotherapies have been shown to be poorly effective with a limited rate of cure observed post-treatment (figure $5 \mathrm{~A}$ and $\mathrm{ref}^{14}$ ) Combination of GAd and anti-PD-1 resulted in a cure rate of $30 \%$ (figure 5A). Addition of BEMPEG to the GAd vaccine was capable of inducing complete regression of tumors in $60 \%$ of the treated animals. Mirroring our findings in the CT26 model, combination of GAd vaccine, anti-PD-1 and BEMPEG increased the rate of response showing complete tumor eradication in $100 \%$ of the mice (figure 5A). These results confirm the therapeutic benefit of this triple combination regimen in a different mouse strain and tumor model. All cured mice rejected a rechallenge with a contralateral tumor inoculum 100 days after the beginning of treatment, confirming the crucial role of the adaptative $\mathrm{T}$ cell response in the cure and the establishment of immunological memory (figure 5B).

\section{DISCUSSION}

Efficacy of anti-PD-1/PDL-1 immune checkpoint inhibitors (CPI) is still limited to a fraction of treated patients. ${ }^{26}$ One of the reasons for this limited success is the fact that CPI act only on some steps of the cancer immunity cycle. Several other drugs including immunomodulators and cancer vaccines are being tested in combination with CPI to improve the rate of response without increasing the adverse events. A recent meta-analysis of the immuneoncology (IO) landscape reported several thousand clinical studies testing IO agents with over one thousand studies involving anti-PD-1/PD-L1 antibodies in combination with other IO agents. ${ }^{26}$ This raises the question of which strategies could be of help to improve the selection of candidates for clinical combination. Understanding 
A
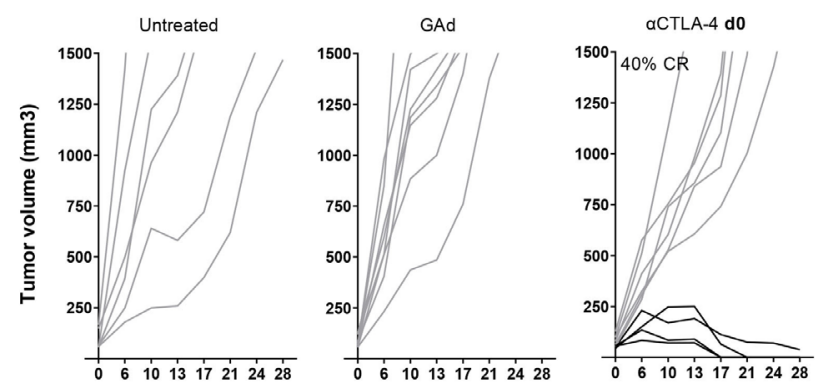

*

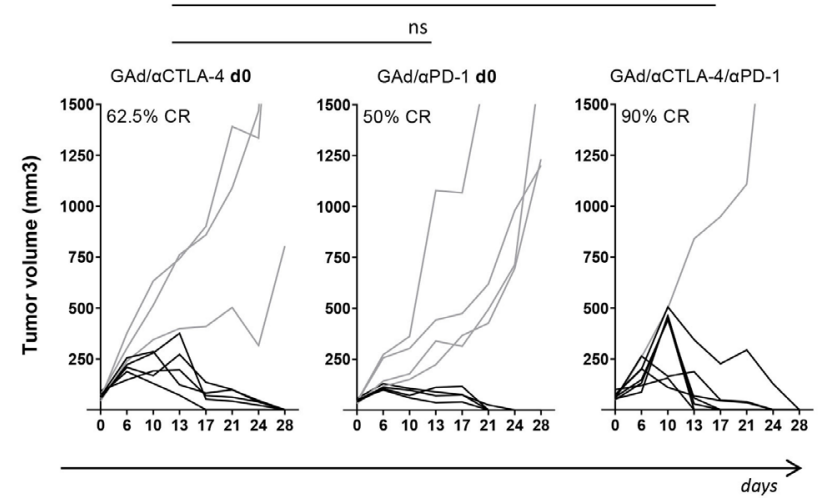

B
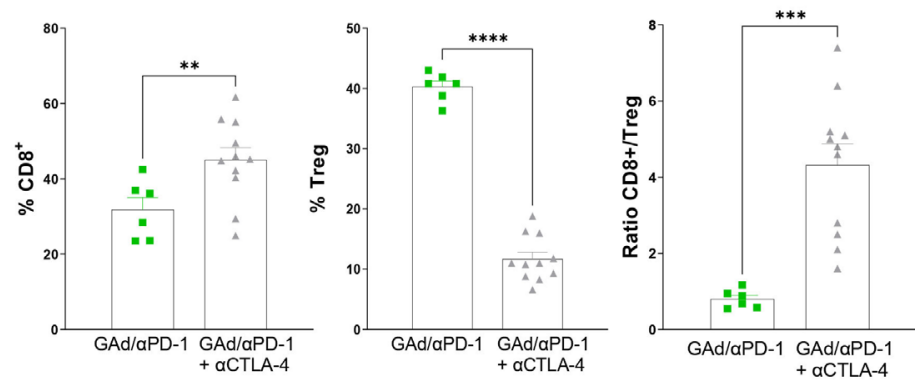

Figure 4 Tumor growth regression is associated with an expansion of intratumor $C D 8^{+} T$ cells and a reduction of intratumor Tregs. (A) BALB/c mice were inoculated subcutaneously with CT26 cells and, after 7 days, they were randomized into six groups: GAd or $\alpha$ CTLA-4 as monotherapies, combo GAd/ $\alpha$ CTLA-4, GAd/ $\alpha$ PD-1 or triple combo GAd/ $\alpha C T L A-4 / \alpha P D-1$. Untreated mice as negative control. Vaccine and $\alpha$ CTLA-4 were administered at do (intramuscular injection, i.m.), and $\alpha P D-$ 1 was given twice per week until d16 (intraperitoneal injection, i.p.). Tumor volume $\left(\mathrm{mm}^{3}\right)$ is shown over time for individual mice. Black curves represent responder mice, gray curves represent non-responder mice. Data ( $\mathrm{n}=6-10$ mice/group) are representative of two independent experiments. Statistics were generated using mid-p exact test $\left({ }^{*} p \leq 0.05\right)$. (B) Intratumor percentages of $\mathrm{CD} 8^{+} \mathrm{T}$ cells or Treg and intratumor $\mathrm{CD} 8^{+} /$Treg ratio are shown for GAd/ $\alpha \mathrm{PD}-1$ (green squares) or GAd/ $\alpha \mathrm{CTLA}-4 /$ $\alpha P D-1$ (gray triangles) treated groups. Bars show mean+SEM. Data ( $n=6-11$ mice/group) were pooled from two independent experiments. Statistics were generated using unpaired Mann-Whitney non-parametric test $\left({ }^{\star \star \star *} p \leq 0.0001,{ }^{* \star *} p \leq 0001,{ }^{* *} p \leq 0.01\right)$. GAd, great apes derived adenovirus.

the mechanism of drug synergy can help in guiding the rational choice of combination drugs to enhance the therapeutic outcome. A series of multiple steps must be initiated and maintained in order to achieve effective killing of tumor cells, which is mediated in large part by $\mathrm{CD}^{+}$cytotoxic $\mathrm{T}$ cells. This cascade of events, referred to as the cancer immunity cycle, shows that there are multiple ways to harness the power of the immune system, and it may be possible to achieve similar results by using different biological tools. Some steps in this circuit of immune regulation are crucial and include: (1) release of tumor antigens, (2) dendritic cells (DCs) maturation and antigen presentation, (3) T cell priming and activation, (4) trafficking of $\mathrm{T}$ cells from the periphery to the tumor, (5) T cell infiltration into the tumor, (6) recognition of the tumor cells by tumor-specific $\mathrm{T}$ cells and (7) killing of tumor cells. Several factors can help in driving or suppressing anticancer immunity at each step of the immunity cycle. In the present work, we made the hypothesis that modulation of key steps of this process can improve the therapeutic response.
Cancer vaccines promote presentation of cancer antigens and priming of tumor selective $\mathrm{T}$ cells in vaccine draining lymph nodes. However, tumor cells use many inhibitory mechanisms to either evade immune system recognition or inhibit the immune response, reducing both the induction and antitumor function of antigenspecific effector $\mathrm{T}$ cells. The expression of immune checkpoint proteins represents one of the many mechanisms of tumor-mediated immunosuppression. The use of anti-PD-1/PD-L1 antibodies to restore the cytotoxic function of tumor-specific $\mathrm{T}$ cells can sustain the activity of vaccine-induced $\mathrm{T}$ cells. Therefore, the combination of a cancer vaccine and immune checkpoint inhibitors may function synergistically to induce more effective antitumor immune responses, and clinical trials to test the combination are currently ongoing. Indeed, we have previously demonstrated and confirmed in the current study that combination of a $\mathrm{nAg}$-based vaccine with checkpoint inhibitors is synergistic and can effectively eradicate large tumors with a cure rate observed in $50 \%$ of treated mice. ${ }^{14}$ We reasoned that the addition 
A
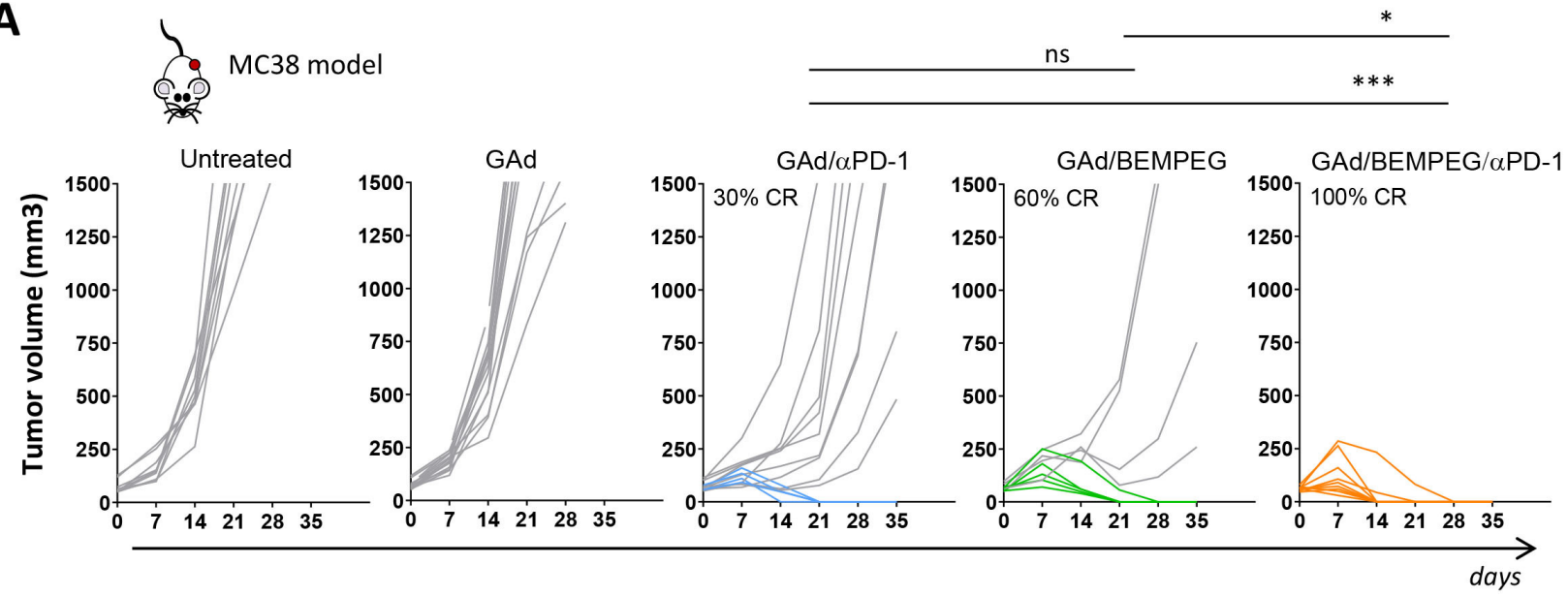

B
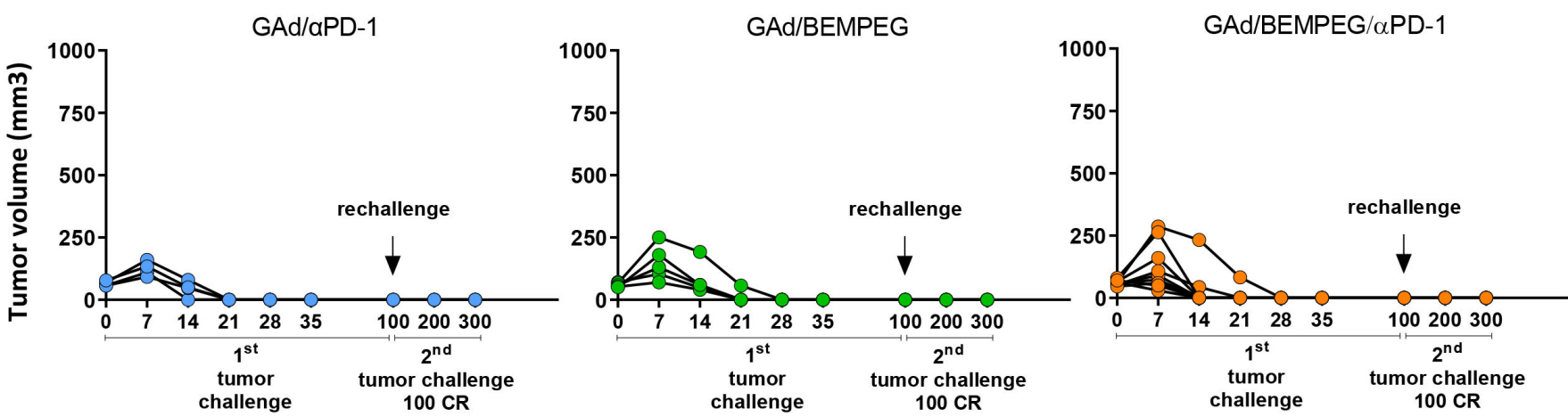

Figure 5 Maximal efficacy of the triple combination GAd/BEMPEG/ 2 PD-1 is confirmed in a second mouse model. (A) C57BL/6 mice bearing 8-day-old subcutaneous MC38 tumors were treated with GAd, combo GAd/ $\alpha$ PD-1, combo GAd/BEMPEG or triple combo GAd/BEMEG/ $\alpha$ PD-1. Tumor volume $\left(\mathrm{mm}^{3}\right)$ was measured over time. Each line represents an individual mouse (gray lines for non-responders and colored lines for responders). Percentages on the graphs indicate the rate of $C R$. Data ( $n=9-13$ mice/group) are representative of two independent experiments. Statistics were generated using mid-p exact test (*** $\leq \leq 0.001$, $\left.{ }^{*} \mathrm{p} \leq 0.05\right)$. (B) Responder mice to GAd/ $\alpha$ PD-1, GAd/BEMPEG or GAd/BEMPEG/ $\alpha$ PD-1 therapy received a second tumor challenge at day 100 . Tumor growth was monitored over time until day 300. CR, complete response; GAd, great apes derived adenovirus.

of a third element modulating the tumor microenvironment and, in particular, the balance between Tregs and $\mathrm{T}$ effector cells at the tumor site, could promote a stronger therapeutic effect. We selected BEMPEG, an investigational CD122-preferential IL-2 pathway agonist with immunomodulatory activity in the tumor microenvironment, as the third component to be added to the combination of GAd and anti-PD-1. Combination of GAd and BEMPEG is also synergistic curing $50 \%$ of treated mice, in line with other evidences demonstrating that IL-2R agonists in conjunction with $\mathrm{nAg}$ vaccine can enhance vaccine-induced $\mathrm{nAg}$-specific $\mathrm{T}$ cells. ${ }^{27} \mathrm{In}$ a preclinical model of large established tumors, we showed that the triple combination of GAd/anti-PD-1/BEMPEG is highly effective and leads to disease eradication in $100 \%$ of treated mice. Addition of BEMPEG to the prior tested therapy resulted in the following superiorities: (1) expansion of vaccine-induced T cells, (2) increased levels of $\mathrm{CD}^{+} \mathrm{T}$ cell infiltration at the tumor site and (3) decrease of the immunosuppressive Treg population.
Transcriptomic analysis of tumors showed that this therapeutic effect of the triple combination corresponded with a dramatic activation of adaptive immunity pathways compared with either double treatment modality. In the triple combination group, BEMPEG specifically induced the upregulation of chemokines and chemokine receptors involved in recruitment of $\mathrm{T}$ cells in the tumor as previously described. ${ }^{23}$ The significant increase of CD8 gene expression in the triple combination confirms the effectiveness of these chemokines in favoring the migration of vaccine-induced $\mathrm{T}$ cells. Moreover, treatment with GAd/anti-PD-1/BEMPEG promoted the induction and maintenance of an effector/activation status of tumor-infiltrating immune cells. One important aspect of our data is the demonstration that effective treatment such as anti-PD-1 or a combination of GAd/anti-PD-1 can induce cytotoxic $\mathrm{T}$ cell activity and can induce an immunosuppressive program that restrains antitumor activity and blunts the response to immunotherapy. A significant increase in the levels of Foxp3 was observed 
in the tumors of mice treated with anti-PD-1 and GAd/ anti-PD-1. BEMPEG drove a striking downregulation of Foxp3, observed in all groups in which BEMPEG was part of the treatment. These results suggest the key contribution of BEMPEG in rebalancing the tumor microenvironment when added to GAd/anti-PD-1 therapy, resulting in an increased therapeutic response. Benefiting from these advantages, the effect of the triple combination in curing $100 \%$ of treated mice was confirmed in a second model.

Combination of BEMPEG with anti-PD-1 is currently being tested in clinical trials with encouraging results indicative of a synergy between CPB and BEMPEG. ${ }^{28}$ Recently, BEMPEG in combination with anti-PD-1 received a breakthrough designation from the United States Food and Drug Administration (FDA) based on improved clinical outcomes in a cohort of patients with metastatic melanoma. This combination showed a favorable safety profile, demonstrating that the addition of BEMPEG did not exacerbate the incidence of AEs typically associated with anti-PD-1 blockade. ${ }^{28}$ The ability of vaccine in boosting pre-existing antitumor response and in inducing de novo $\mathrm{T}$ cell response holds great potential in contributing to the efficacy of anti-PD-1 and BEMPEG combination. Moreover, the vaccine platform presented here allows the inclusion of multiple neoepitopes in tandem thereby increasing the chances of triggering relevant $T$ cell responses to improve the antitumor efficacy. Indeed, one of the unique features of the GAd technology is the capability to accommodate large gene inserts, encoding many nAgs. In a clinical setting, a cancer vaccination targeting nAgs is, in most cases, a personalized approach and requires selection of mutations with a high likelihood to behave as nAgs based on predictive algorithms. Therefore, the possibility to include many nAgs has a significant advantage by increasing the probability of having, among the selected ones, both those already spontaneously immunogenic and those that can induce effective de novo responses. In this context, the addition of vaccine to anti-PD-1 and BEMPEG is expected to be safe, being very selective to antigens that are present exclusively in the tumor. Moreover, preliminary data on the combination of nAg-based vaccine with pembrolizumab in metastatic patients with microsatellite instability tumors showed a safe and well-tolerated profile (NCT04041310 $0^{29}$ ). Aiming to corroborate the mechanism behind the maximal antitumor efficacy of GAd, anti-PD-1 and BEMPEG, we employed a different therapeutic approach selecting as the tumor modulating agent, an anti-CTLA-4 Ab with Treg-depleting activity. Triple combination of GAd, antiPD-1 and anti-CTLA-4 recapitulated the maximal efficacy of GAd, anti-PD-1 and BEMPEG in the CT26 model with the vast majority of treated mice cured post-triple therapy, as compared with single and dual treatment regimens. Also in this case, the addition of anti-CTLA- 4 to the dual therapy, GAd and anti-PD-1, promoted a change in the tumor microenvironment in favor of $\mathrm{CD}^{+} \mathrm{T}$ cells over
Tregs, underlying the ratio of intratumoral Teff/Treg as a relevant biomarker of treatment efficacy.

Overall, data suggest that tumor microenvironment reprogramming is crucial to potentiate $\mathrm{nAg}$ vaccination and checkpoint blockade therapy and could be a possible cornerstone to potentiate antitumor immunity and efficacy in a safe and effective way. These findings elucidate essential characteristics of combination immunotherapies underlying that rejection of established tumors in all treated mice relied on an orchestrated response invoking diverse mechanisms of the immune system.

Acknowledgements We would like to thank Marina Udier for critical reading of the manuscript and helpful discussion. We acknowledge the animal facility of Plaisant in Castel Romano (Rome) for maintenance and care of the mice used in this study. We would like to thank in particular Domenico Salvatori for his support with tumor calibrations for in vivo studies. We would like to acknowledge Maria Ravo and Francesca Rizzo for their technical support with NGS data.

Contributors ES, AN, and AMD'A designed the study. AMD'A, GL, GC, FT, FL, MDL, IG, LN, RV, EDM, VB, RB, FGT, and EDM conducted the research. ES, AMD, and GL analyzed and interpreted the data. JZ and DHC contributed to interpreted the data. AL contributed to NGS data interpretation. ES, AMD'A, GL, AN, and JZ wrote the manuscript. ES was the principal investigator and acts as guarantor.

Funding The authors have not declared a specific grant for this research from any funding agency in the public, commercial or not-for-profit sectors.

Competing interests ES and AN are founders of Nouscom. JZ and DHC are current or past employees and shareholders of Nektar Therapeutics. The remaining authors are employees of Nouscom.

Patient consent for publication Not applicable.

Ethics approval All the in vivo experimental procedures were approved by the local animal ethics council and performed in accordance with national and international laws and policies (EEC Council Directive 86/609; Italian Legislative Decree 26/14). The ethical committee of the Italian Ministry of Health approved this research.

Provenance and peer review Not commissioned; externally peer reviewed.

Data availability statement Data are available on reasonable request. All data relevant to the study are included in the article or uploaded as supplementary information.

Open access This is an open access article distributed in accordance with the Creative Commons Attribution Non Commercial (CC BY-NC 4.0) license, which permits others to distribute, remix, adapt, build upon this work non-commercially, and license their derivative works on different terms, provided the original work is properly cited, appropriate credit is given, any changes made indicated, and the use is non-commercial. See http://creativecommons.org/licenses/by-nc/4.0/.

\section{ORCID iD}

Anna Morena D'Alise http://orcid.org/0000-0002-0763-6269

\section{REFERENCES}

1 Rosenberg SA. IL-2: the first effective immunotherapy for human cancer. J Immunol 2014;192:5451-8.

2 Boyman O, Sprent J. The role of interleukin-2 during homeostasis and activation of the immune system. Nat Rev Immunol 2012;12:180-90.

3 Bentebibel S-E, Hurwitz ME, Bernatchez C, et al. A first-in-human study and biomarker analysis of NKTR-214, a novel IL2R $\beta \gamma$-Biased cytokine, in patients with advanced or metastatic solid tumors. Cancer Discov 2019;9:711-21.

4 Charych DH, Hoch U, Langowski JL, et al. NKTR-214, an engineered cytokine with biased IL2 receptor binding, increased tumor exposure, and marked efficacy in mouse tumor models. Clin Cancer Res 2016;22:680-90.

5 Charych D, Khalili S, Dixit V, et al. Modeling the receptor pharmacology, pharmacokinetics, and pharmacodynamics of NKTR-214, a kinetically-controlled interleukin-2 (IL2) receptor agonist for cancer immunotherapy. PLoS One 2017;12:e0179431. 
6 Sharma M, Khong H, Fa'ak F, et al. Bempegaldesleukin selectively depletes intratumoral Tregs and potentiates T cell-mediated cancer therapy. Nat Commun 2020;11:661.

7 Sharma P, Allison JP. The future of immune checkpoint therapy. Science 2015;348:56-61.

8 Ribas A, Wolchok JD. Cancer immunotherapy using checkpoint blockade. Science 2018;359:1350-5.

9 Gellrich F, Schmitz M, Beissert S, et al. Anti-PD-1 and nove combinations in the treatment of Melanoma-An update. J Clin Med 2020;9:223

10 Kreiter S, Vormehr M, van de Roemer N, et al. Mutant MHC class II epitopes drive therapeutic immune responses to cancer. Nature 2015;520:692-6.

11 Yadav M, Jhunjhunwala S, Phung QT, et al. Predicting immunogenic tumour mutations by combining mass spectrometry and exome sequencing. Nature 2014;515:572-6.

12 Sahin U, Derhovanessian E, Miller M, et al. Personalized RNA mutanome vaccines mobilize poly-specific therapeutic immunity against cancer. Nature 2017;547:222-6.

13 Ott PA, Hu Z, Keskin DB, et al. An immunogenic personal neoantigen vaccine for patients with melanoma. Nature 2017;547:217-21.

14 D'Alise AM, Leoni G, Cotugno G, et al. Adenoviral vaccine targeting multiple neoantigens as strategy to eradicate large tumors combined with checkpoint blockade. Nat Commun 2019;10:2688.

15 Tatsis N, Ertl HCJ. Adenoviruses as vaccine vectors. Mol Ther 2004;10:616-29.

16 Capone S, D'Alise AM, Ammendola V, et al. Development of chimpanzee adenoviruses as vaccine vectors: challenges and successes emerging from clinical trials. Expert Rev Vaccines 2013;12:379-93.

17 Barnes E, Folgori A, Capone S, et al. Novel adenovirus-based vaccines induce broad and sustained $\mathrm{T}$ cell responses to HCV in man. Sci Trans/ Med 2012;4:115ra1.

18 Swadling L, Capone S, Antrobus RD, et al. A human vaccine strategy based on chimpanzee adenoviral and MVA vectors that primes, boosts, and sustains functional HCV-specific T cell memory. Sci Trans/ Med 2014:6:261ra153.

19 O'Hara GA, Duncan CJA, Ewer KJ, et al. Clinical assessment of a recombinant simian adenovirus ChAd63: a potent new vaccine vector. J Infect Dis 2012;205:772-81.

20 Green CA, Scarselli E, Sande CJ, et al. Chimpanzee adenovirusand MVA-vectored respiratory syncytial virus vaccine is safe and immunogenic in adults. Sci Transl Med 2015;7:300ra126.

21 Blattman JN, Grayson JM, Wherry EJ, et al. Therapeutic use of IL-2 to enhance antiviral T-cell responses in vivo. Nat Med 2003;9:540-7.

22 Ayers M, Lunceford J, Nebozhyn M, et al. IFN-gamma-related mRNA profile predicts clinical response to PD-1 blockade. J Clin Invest 2017;127:2930-40.

23 Danaher P, Warren S, Lu R. Pan-Cancer adaptive immune resistance as defined by the tumor inflammation signature (TIS): results from the cancer genome atlas (TCGA). J Immunother Cancer 2018;6:63.

24 Harlin H, Meng Y, Peterson AC, et al. Chemokine expression in melanoma metastases associated with $\mathrm{CD}^{+} \mathrm{T}$-cell recruitment. Cancer Res 2009;69:3077-85

25 Selby MJ, Engelhardt JJ, Quigley M, et al. Anti-CTLA-4 antibodies of IgG2a isotype enhance antitumor activity through reduction of intratumoral regulatory T cells. Cancer Immunol Res 2013;1:32-42.

26 Tang J, Shalabi A, Hubbard-Lucey VM. Comprehensive analysis of the clinical immuno-oncology landscape. Ann Oncol 2018;29:84-91.

27 Hernandez R, LaPorte KM, Hsiung S, et al. High-dose IL-2/ CD25 fusion protein amplifies vaccine-induced CD4 ${ }^{+}$and CD8 ${ }^{+}$neoantigen-specific $T$ cells to promote antitumor immunity. $J$ Immunother Cancer 2021;9:e002865.

28 Diab A, Tykodi SS, Daniels GA, et al. Bempegaldesleukin plus nivolumab in first-line metastatic melanoma. JCO 2021;39:2914-25.

29 Leoni G, D'Alise AM, Cotugno G, et al. A genetic vaccine encoding shared cancer neoantigens to treat tumors with microsatellite instability. Cancer Res 2020;80:3972-82. 